\title{
Scattering of the Fundamental Symmetrical Lamb Wave Mode by a Small Edge Crack in an Isotropic Plate
}

\author{
Benjamin Steven Vien ${ }^{1}$, Nithurshan Nadarajah ${ }^{1}$, Wing Kong Chiu ${ }^{1}$ and L. R. Francis Rose ${ }^{2}$ \\ 1. Department of Mechanical and Aerospace Engineering, Monash University, Clayton 3800, Australia \\ 2. Defence Science \& Technology Organisation, Aerospace Division, Port Melbourne 3207, Australia
}

Received: March 23, 2015 / Accepted: April 07, 2015 / Published: April 25, 2015.

\begin{abstract}
Lamb waves have a relatively slow geometrical decay of amplitude with propagation distance, which makes them well suited for wide-area inspection and SHM (structural health monitoring). This paper presents an extensive computational study of the interaction of the fundamental symmetrical Lamb wave mode (S0) with a through-thickness edge crack in an isotropic plate. The incident wave is generated by a point source that is symmetrical with respect to the plate's midplane, and whose time dependence is a 10-cycle Hanned windowed toneburst of centre frequency below the cut-off for the first order shear-horizontal mode (SH1), so that the only propagating modes are S0 and SH0. Results are presented showing the angular dependence of the S0 and SH0 scattered fields for various angles of incidence. The dependence of scattering amplitude on crack size is also studied, with a view to facilitating the inverse problem of determining crack size from measurements of the scattered field. It is shown that the scattered field due to a small crack can be considered to be equivalent to a point source consisting of a combination of force doublets that depend on the angle of incidence. The implications of these results for SHM are briefly discussed.
\end{abstract}

Key words: Lamb waves, finite element modelling, fatigue cracks, structural health monitoring.

\section{Introduction}

Fatigue cracks in engineering structures are a common problem and require regular inspections to detect and monitor small cracks before they reach a critical size that could result in catastrophic failure. Conventional ultrasonic techniques, such as pulse-echo and pitch-catch method [1], involve scanning over the area of interest and require local accessibility for inspections. This conventional technique is very time consuming and can be impractical for crack detection in large and complex structures. Lamb waves propagation is an alternative method which has been acknowledged by many authors for ultrasonic non-destructive damage detection due to its attractive properties for wide-area inspection with little attenuation. Lamb waves and

Corresponding author: Benjamin Steven Vien, Ph.D., research field: structural health monitoring. E-mail: ben.vien@monash.edu. their applications have been extensively investigated, both computationally and experimentally $[2-8,12,13$, 17-19]. The FE (finite element) method provides a time-efficient and advantageous computational tool to analyze wave propagation in complicated structures and configurations for which the analytical solutions do not exist.

The scattered wave fields when an incident elastic wave impinges on a crack have been investigated for bulk waves [9-11], and more recently, for Lamb waves $[12,13]$. It is known that an infinitesimal crack is equivalent to a point source consisting of body-force doublets with different forcing combinations that correspond to different crack opening modes [14]. This suggests that the scattered wave field due to the presence of small edge crack can be modelled as point source when the incident wavelength is much longer than the crack dimension.

The aim of this paper is to computationally explore the interaction of low frequency zeroth order 
symmetrical modes with a small (compared to wavelength) edge crack. The first investigation will report on the scattered wave pattern and amplitude for various angles of incidence and various crack lengths. The second set of investigation is to simulate the scattered wave pattern by applying tractions on the crack faces to enforce a traction-free boundary condition, based on the principle of superposition. The motivation for this study is to investigate and analyze the scattered wave field from a known crack size as a pre-requisite for a subsequent attempt to investigate the inverse problem of characterizing the crack size based on the measurements of the scattered field. The nature of the wave scattered by an edge crack will be reported on.

\section{Computational Set-up and Procedure}

Lamb waves are generally dispersive and if excited at higher frequency-thickness product, multiple modes will exist and the acquired signals may be difficult to interpret. However, if excited by a point source that is symmetrical with respect to the plate's midplane and at low frequency-thickness product range, below the cut-off of $1.53 \mathrm{MHz}-\mathrm{mm}$ for aluminium [15], the Lamb waves consist of only two propagating modes, viz. the fundamental symmetric wave (S0) and shear horizontal wave (SH0). Previous studies $[12,13]$ have considered SH0 Lamb wave propagation due to its non-dispersive properties, however, experimentally exciting only the SH0 wave is difficult compared to exciting S0. In this study, only S0 Lamb wave excitation will be considered.

A baseline subtraction is employed to separate the scattered wave displacement field, $u_{\text {scatter }}$, associated with the small edge cracks as follows (Fig. 1):

$$
u_{\text {scatter }}(r, \theta, t)=u_{\text {total }}(r, \theta, t)-u_{\text {baseline }}(r, \theta, t)
$$

where, $u_{\text {total }}$ denotes the response of the cracked structure, whereas $u_{\text {baseline }}$ denotes the baseline field that is generated by the same excitation but in the absence of a defect.

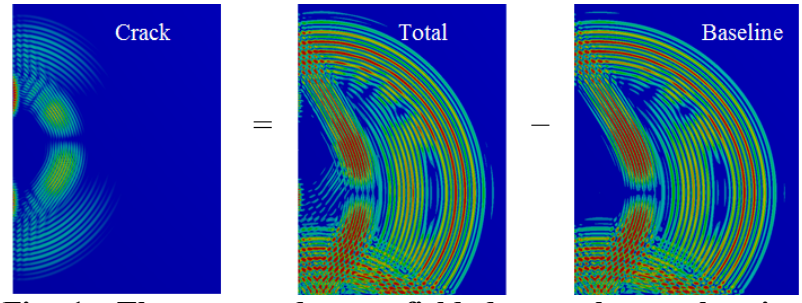

Fig. 1 The scattered wave field due to the crack using baseline subtraction from Eq. (1).

(a)

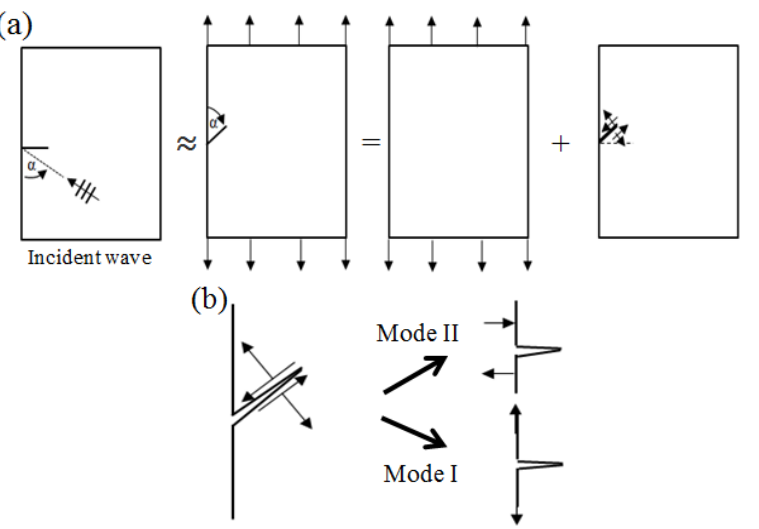

Fig. 2 (a) Wave field as a representation of static stress approximation and (b) scattered wave by the edge crack is equivalent to superposition of Mode I and II crack opening displacements response.

The scattered field can also be obtained as the field generated by applying equal and opposite tractions on the crack faces that cancel those produced by the incident S0, in accordance with the principle of superposition [16-18], as illustrated in Fig. 2. This will meet the condition of a traction-free crack after the superposition of the incident and scattered field. For this purpose, only the baseline $\sigma_{x x}$ and $\sigma_{x y}$ stresses are required as any variant in the normal direction, $z$, is negligible due to the thin-plate approximation. Since the crack is sufficiently small, the stresses along the crack face are approximately constant along the crack, and hence only one of nodal stresses is required. The time sequence of each baseline stresses are then applied as tractions on the $3 \mathrm{~mm}$ and $6 \mathrm{~mm}$ crack faces to generate the wave fields that correspond to the scattered fields obtained from Eq. (1).

\section{Computational Investigation}

ANSYS 14.0 is used as the FE computational 
analysis tool for this study. The simulation represents an aluminium test plate $800 \mathrm{~mm} \times 600 \mathrm{~mm} \times 1 \mathrm{~mm}$ (density of $2,700 \mathrm{~kg} / \mathrm{m}^{3}$, Poisson's ratio of 0.33 and Young modulus of $69 \mathrm{GPa}$ ) as shown in Fig. 3a. The plate contains an edge crack located at the origin, as indicated in Fig. 3. The plate is discretized into 8-node linear cubic elements of size $1 \mathrm{~mm}$, and the crack is accordingly modelled as a notch of width equal to 1 $\mathrm{mm}$, which is much smaller than the crack length, $a$, of $3 \mathrm{~mm}$ and $6 \mathrm{~mm}$ for the angular dependence investigation. The maximum amplitude of the scattered S0 and SH0 wave pattern at $45^{\circ}$ incidence is also investigated for crack lengths from $1 \mathrm{~mm}$ to 9 $\mathrm{mm}$ with $1 \mathrm{~mm}$ increment, to determine the dependence of scattered amplitude with crack length.

The incident S0 mode is generated by applying equal and opposite point forces to nodes on opposite faces of the plate, with the force acting in the direction normal to surface, which can be aptly described as a pinching force. The pinching force excitation signal consisted of a 10-cycle Hanned window tone burst centred at frequency $200 \mathrm{kHz}$ to minimize dispersion. At this centre frequency, the incident S0 mode has a wavelength $\lambda_{\mathrm{S} 0}=26.6 \mathrm{~mm}$, and SH0 mode has a wavelength $\lambda_{\mathrm{SH} 0}=15.3 \mathrm{~mm}$. This input excitation is applied at point $\mathrm{P}$ with polar coordinates $\left(R_{1}, \theta^{I}\right)$. To approximate a planar incident wave, the cylindrical excitation source is placed at $5 \lambda_{\mathrm{S} 0}$ away, to ensure that the impinging wavelength at $\lambda_{\mathrm{S} 0} / 2$ along the boundary from the crack base is no more than $0.05 \lambda_{\mathrm{S} 0}$ in error compared with a plane wave. Polar coordinates $(r, \theta)$ with origin at the crack base and the $r$ and $\theta$ components of the surface displacement will be used to track the scattered Lamb wave modes (Fig. 3).

The cracks are modelled as a $1 \mathrm{~mm}$ width notch by element removal in FE. The notch width is sufficiently large to prevent contact from the adjacent crack face and small enough to approximate to a zero-width discontinuity [8] such that reflectivity from notch width is negligible.

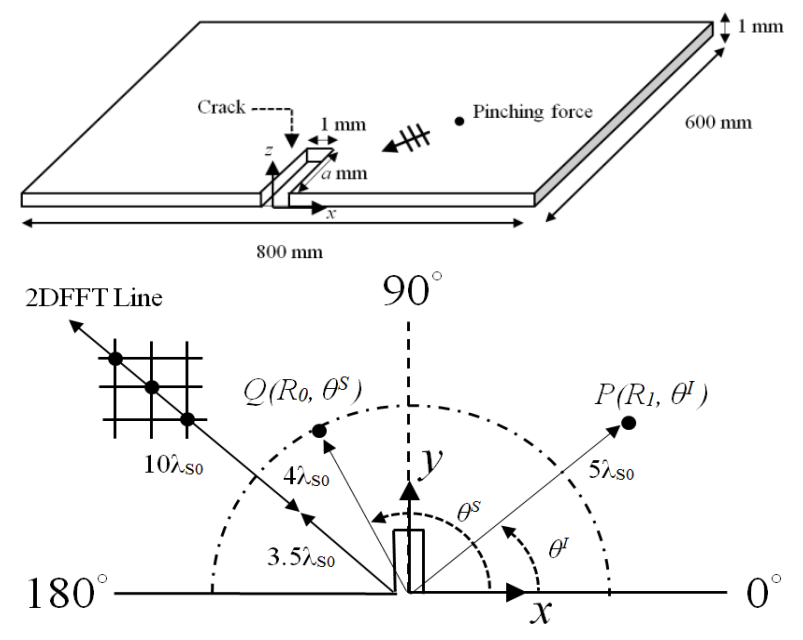

Fig. 3 (a) Geometry of FE model for $800 \mathrm{~mm} \times 600 \mathrm{~mm} \times$ $1 \mathrm{~mm}$ aluminium plate showing the location of the excited force relative to the crack and (b) detail diagram showing the 2D FFT line, point of excitation and scattered wave measurement locations relative to the crack.

The FE simulation uses $1 \mathrm{~mm}$ cubic element size, which satisfies the requirement of 10 elements per wavelength for accurate modeling [19], and a time step of $0.1 \mu \mathrm{s}$, which satisfies the explicit time integration $0.8 \mathrm{~L} / \mathrm{C}$ stability limit [20], where $\mathrm{L}$ is the smallest element length, $\mathrm{C}$ is the fastest wave speed. A 2D FFT (fast Fourier transform) is performed on the nodes along the line $45^{\circ}$ from the crack base edge indicated in Fig. $3 \mathrm{~b}$ and was used to produce the dispersion curves to identify the dominant scattered Lamb wave mode in the $\theta$ and $r$ components from Ref. [15], as shown in Figs. $4 \mathrm{a}$ and $4 \mathrm{~b}$. The $2 \mathrm{D}$ FFT consists of more than 400 time samples, $40 \mu \mathrm{s}$. The 2D FFT spatial distance is taken at least $3.5 \lambda_{\mathrm{S} 0}$ away from the crack base, to avoid detection of higher non-propagating Lamb wave modes [18] and over approximately $10 \lambda_{\mathrm{S} 0}$ distances with 1,024 equidistant spatial samples with zero padding [19]. The scattered wave pattern is obtained by polar plotting the maximum displacement values, which is obtained by performing a Hilbert transformation over a time-domain [1], measured at point $\mathrm{Q}$ with polar coordinates $\left(R_{0}, \theta^{S}\right)$ at distance $R_{0} \approx 4 \lambda_{\mathrm{S} 0}$ away as shown in Fig. 3b.

The scattered patterns are analyzed in two sections: 

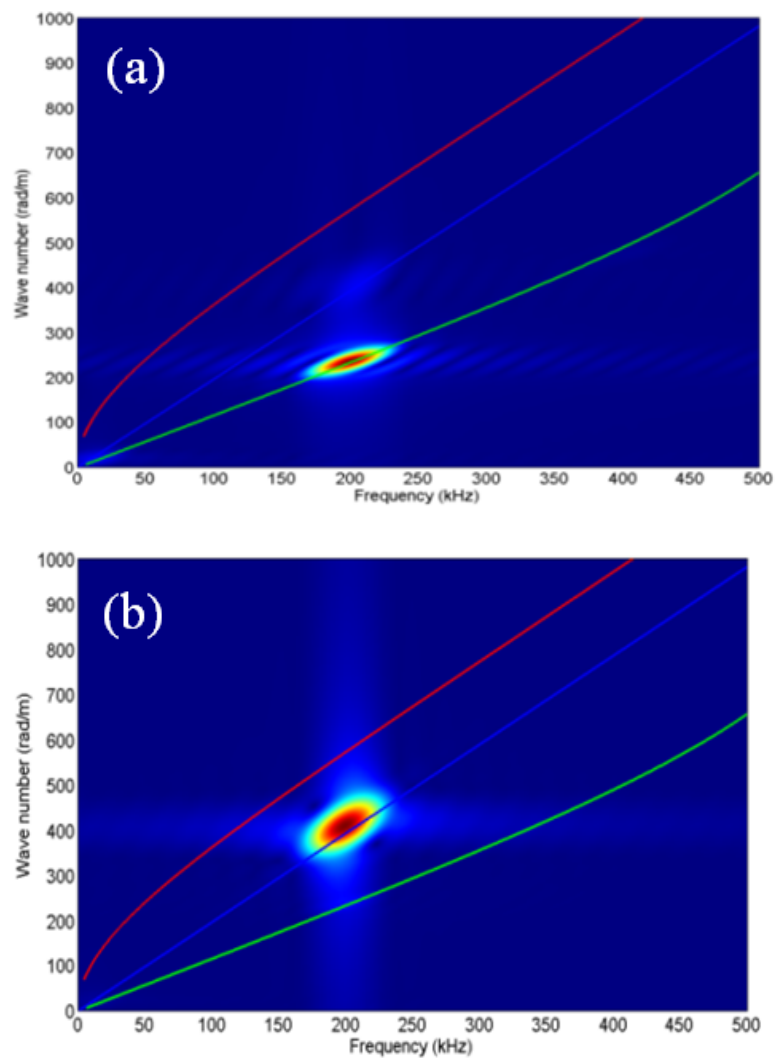

Fig. 4 (a) Dispersion curve for scattered wave field of 3 mm edge crack in the radial component with dominant SO and (b) dispersion curve for scattered wave field of $3 \mathbf{~ m m}$ edge crack in the $\theta$ component with dominant SH0: AO(red), SHO(blue) and SO(green).

back-scattered displacement, $u_{B}$, measured in $0^{\circ} \leq \theta<$ $90^{\circ}$ and forward-scattered displacement, $u_{F}$, as measured in $90^{\circ} \leq \theta \leq 180^{\circ}$. The maximum scattered wave, $u_{\max }$, relative to maximum input excitation displacement as the reference displacement, $u_{\text {ref }}$, in relation to angle dependence and crack length dependence are investigated. The scattered field maximum displacement is considered to normalize the scattered wave field in order to observe the scattered displacements relative to its maxima. The edge wave displacements are disregarded because the purpose is simply to compare the $\mathrm{S} 0$ and $\mathrm{SH} 0$ scattering patterns without considering the absolute values of amplitude. The scattered wave pattern for various crack lengths are investigated and later compared in the second investigation to the wave patterns produced by applying tractions on the crack faces.

\section{Results}

4.1 Scattered Field for Various Incident Angles and Various Crack Lengths

The investigation includes results of scattered wave pattern generated by an S0 Lamb wave at various incident angles to an edge crack of length $3 \mathrm{~mm}(a / \lambda=$ $0.11)$ and $6 \mathrm{~mm}(a / \lambda=0.23)$. The dispersion curves have indicated predominant scattered SH0 and S0 Lamb wave in the $\theta$ and $r$ components, respectively. The results also indicated the propagation of Rayleigh-like edge waves. In general, the larger $a / \lambda$ scattered amplitude have stronger S0, SH0 and edge wave scattered maximum displacement and the scattered SH0 waves are relatively stronger compared to the scattered S0 waves, as can be seen in Figs. 7 and 8 .

The increase of crack length has shown an increase of forward-scattered lobe as shown in Fig. 9 and a trend of increasing maximum displacement value as shown in Fig. 10. It can be seen that the maximum scattered displacement is approximately proportional to $a / \lambda$ until $a / \lambda \approx 0.23$, beyond which the maximum displacement values appear to plateau.

The $\theta$ component results of $a / \lambda=0.11$ and 0.23 scattered wave patterns, refer to Figs. 11 and 14, showed that as incident angle decreases or crack length increase the maximum scattered amplitude increases. However, the SH0 scattered wave patterns are distinct at different incident angles. At $75^{\circ}$ and $60^{\circ}$ incidences, in Figs. 12 and 15, there is a larger forward-scattered lobe which is almost symmetrical at the $90^{\circ}$ line. The forward and back-scattered amplitude ratio approaches to unity when incident angle is small. Furthermore, the $a / \lambda=0.11$ and 0.23 SH0 wave pattern appears more symmetrical along the $90^{\circ}$ line and similar to a SH0 point source for smaller angle of incidence, which can be seen in Figs. 12d and $15 \mathrm{~d}$. The symmetrical wave pattern indicates dominant primary forcing component, either perpendicular or parallel, acting on the crack at the extreme angle of 

Small Edge Crack in an Isotropic Plate
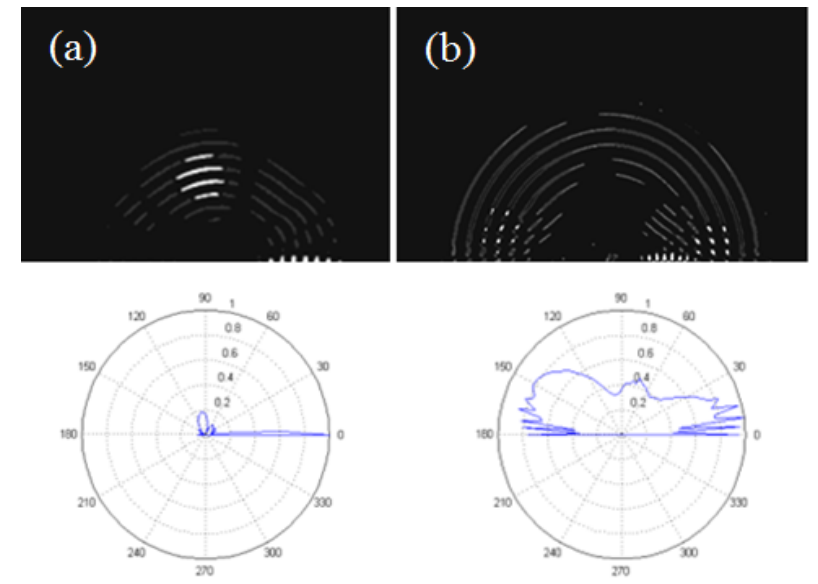

Fig. 5 Scattered wave pattern and polar plot of $3 \mathrm{~mm}$ crack, $a / \lambda=0.11$, at incident $60^{\circ}$ (a) $\theta$ component and (b) radial component.

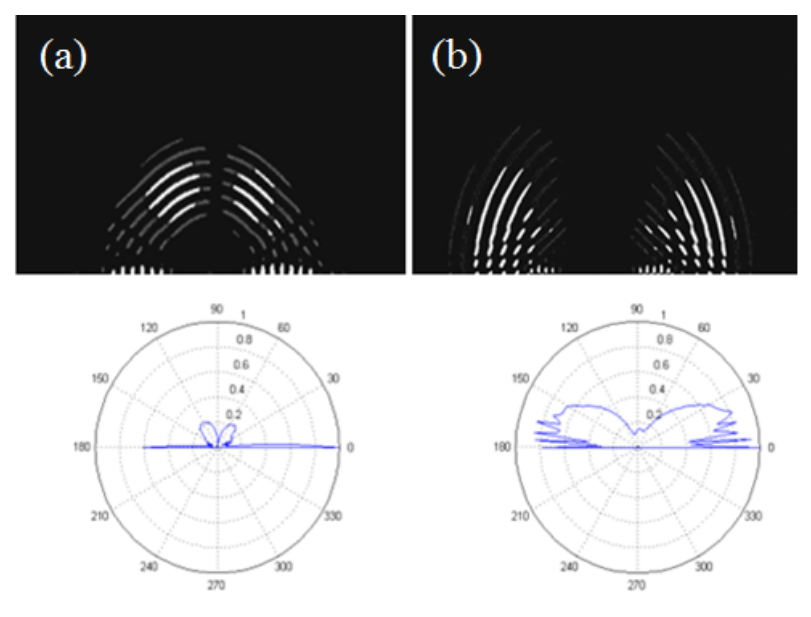

Fig. 6 Scattered wave pattern and polar plot of $3 \mathrm{~mm}$ crack, $a / \lambda=0.11$, at incident $30^{\circ}$ (a) $\theta$ component and (b) radial component.

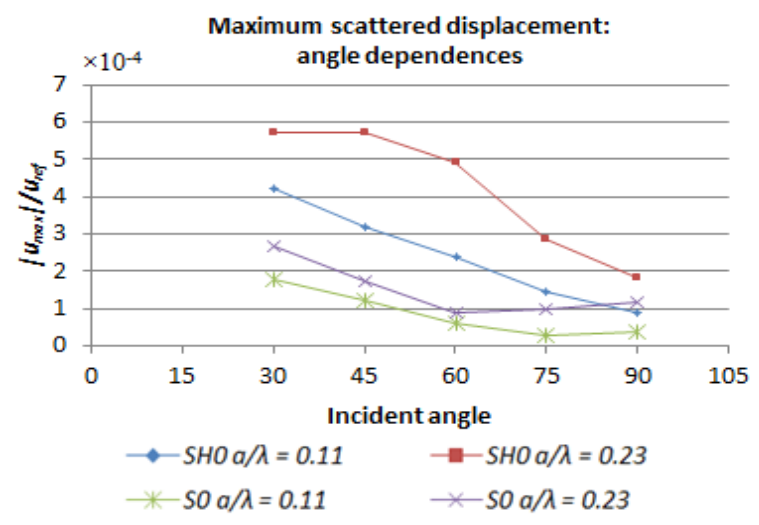

Fig. 7 Graph of the maximum value of SHO and SO scattered displacement with various incident angles for $a / \lambda$ $=0.11$ and 0.23 .

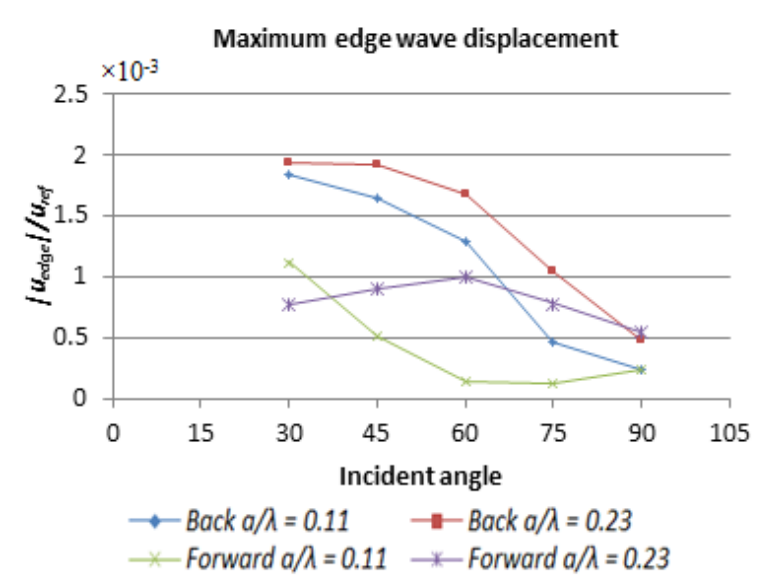

Fig. 8 Graph of the maximum forward and back-scattered edge wave displacement with various incident angle for $a / \lambda=0.11$ and 0.23 .

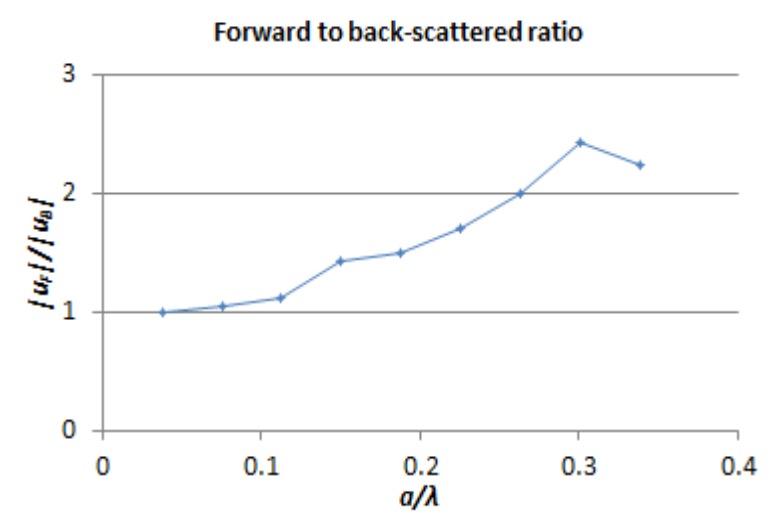

Fig. 9 Maximum forward to back-scattered displacement ratio of scattered waves at incident $45^{\circ}$ with various $a / \lambda$.

Maximum scattered displacement: crack length dependences

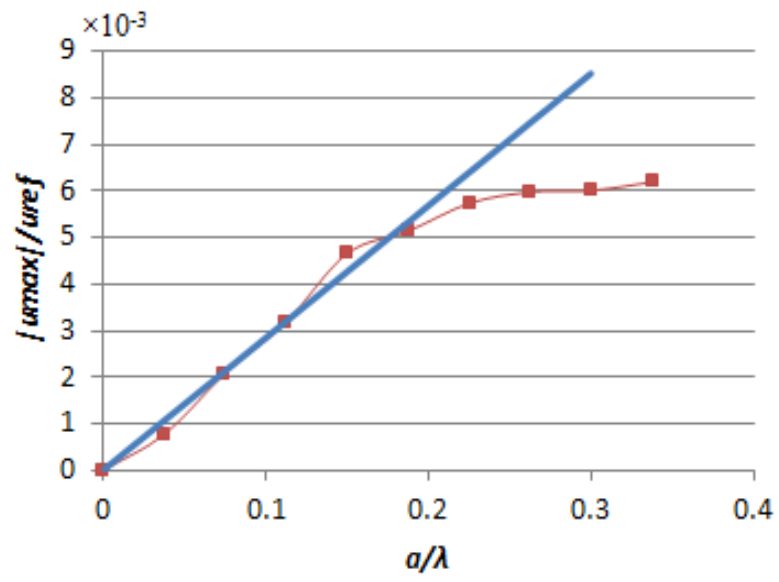

Fig. 10 Graph of the maximum scattered displacement at incident $45^{\circ}$ with various $a / \lambda$. 


\section{Small Edge Crack in an Isotropic Plate}

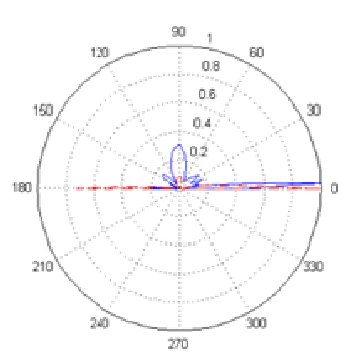

(a)

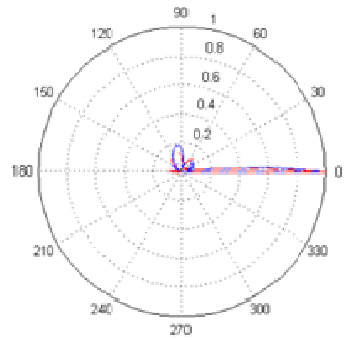

(b)

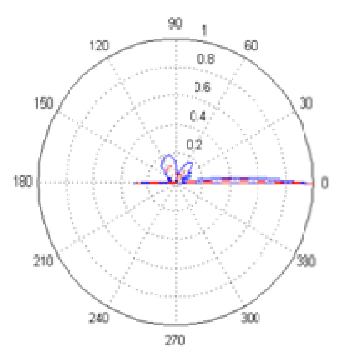

(c)

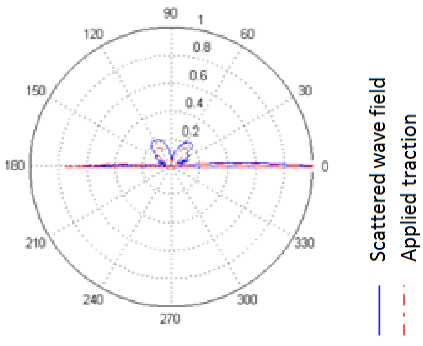

(d)

Fig. 11 [a-d] $\theta$ component $a / \lambda=0.11(3 \mathrm{~mm})$ crack: comparison of applied-traction to original SH0 scattered wave patterns, normalized with maximum scattered for $75^{\circ}, 60^{\circ}, 45^{\circ}$ and $30^{\circ}$ incidences, respectively.

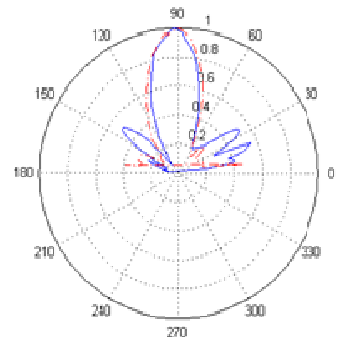

(a)

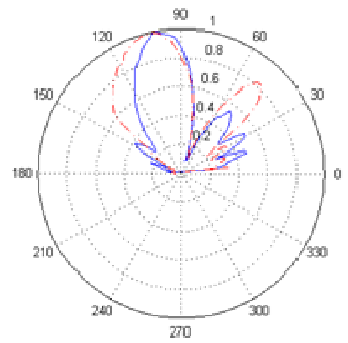

(b)

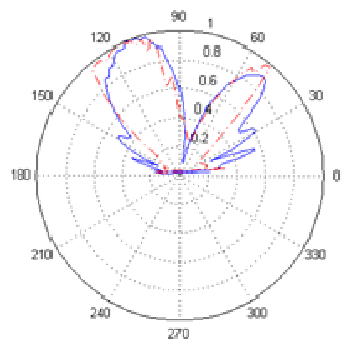

(c)

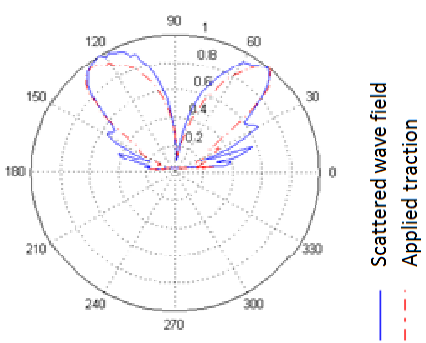

(d)

Fig. 12 [a-d] $\theta$ component $a / \lambda=0.11(3 \mathrm{~mm})$ crack: comparison of applied-traction to original SHO scattered wave patterns, normalized without edge waves for $75^{\circ}, 60^{\circ}, 45^{\circ}$ and $30^{\circ}$ incidences, respectively.

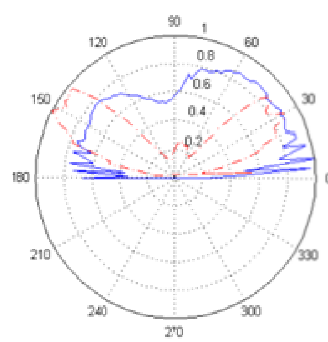

(a)

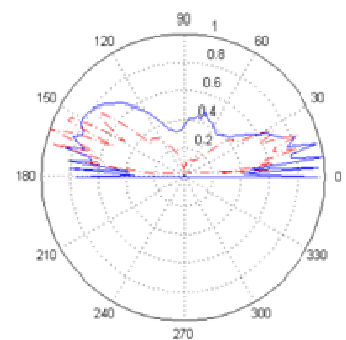

(b)

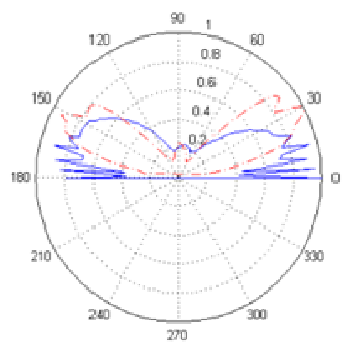

(c)

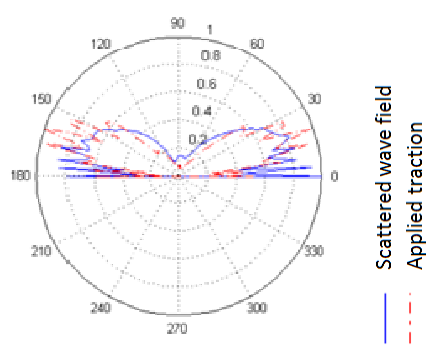

(d)

Fig. 13 [a-d] Radial component $a / \lambda=0.11(3 \mathrm{~mm})$ crack: comparison of applied-traction to original S0 scattered wave patterns, normalized without edge waves for $75^{\circ}, 60^{\circ}, 45^{\circ}$ and $30^{\circ}$ incidences, respectively.

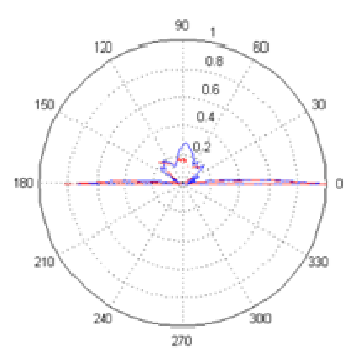

(a)

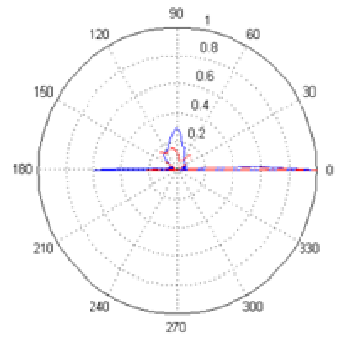

(b)

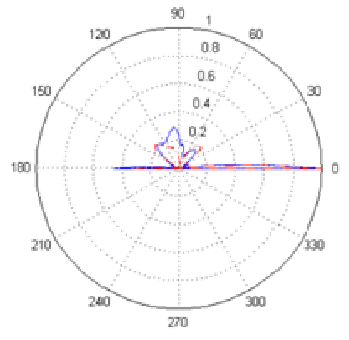

(c)

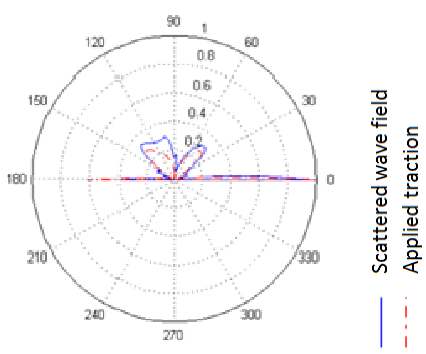

(d)

Fig. 14 [a-d] $\theta$ component $a / \lambda=0.23(6 \mathrm{~mm})$ crack: comparison of applied-traction to original SH0 scattered wave patterns, normalized with maximum scattered for $75^{\circ}, 60^{\circ}, 45^{\circ}$ and $30^{\circ}$ incidences, respectively. 


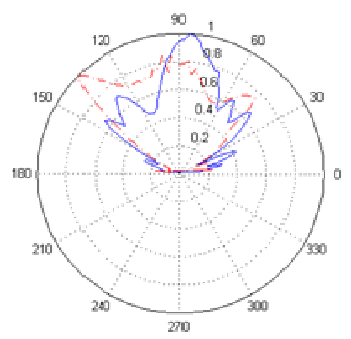

(a)

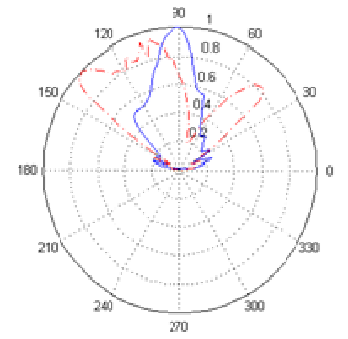

(b)

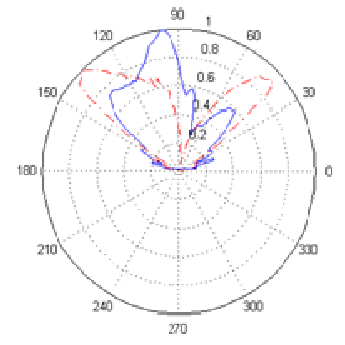

(c)

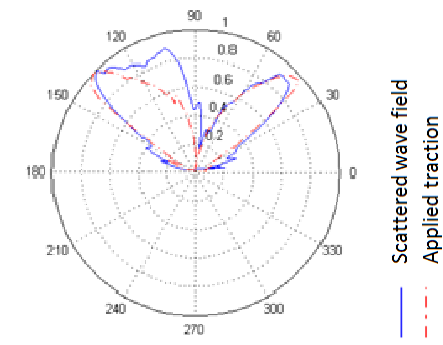

(d)

Fig. 15 [a-d] $\theta$ component $a / \lambda=0.23(6 \mathrm{~mm})$ crack: comparison of applied-traction to original SHO scattered wave patterns, normalized without edge waves for $75^{\circ}, 60^{\circ}, 45^{\circ}$ and $30^{\circ}$ incidences, respectively.

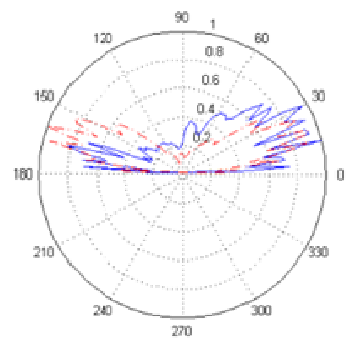

(a)

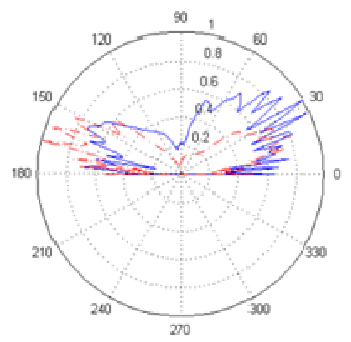

(b)

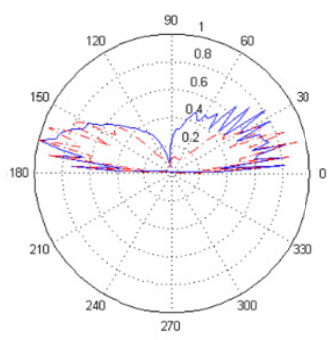

(c)

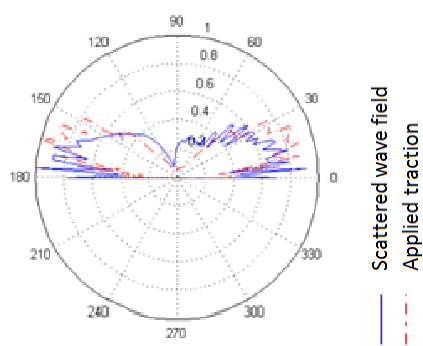

(d)

Fig. 16 [a-d] Radial component $a / \lambda=0.23(6 \mathrm{~mm})$ crack: Comparison of applied-traction to original S0 scattered wave patterns, normalized without edge waves for $75^{\circ}, 60^{\circ}, 45^{\circ}$ and $30^{\circ}$ incidences, respectively.

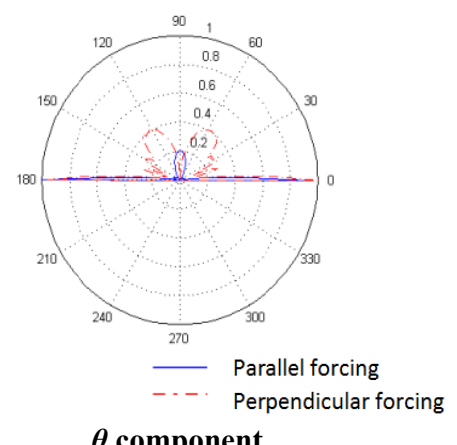

$\theta$ component

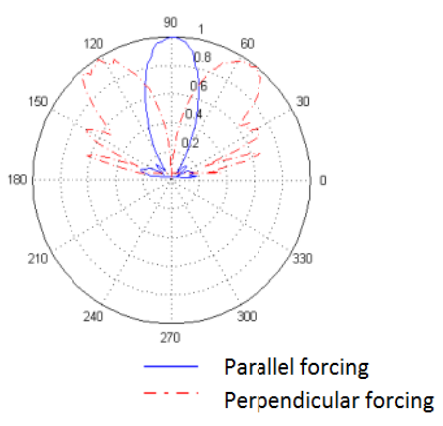

$\theta$ component

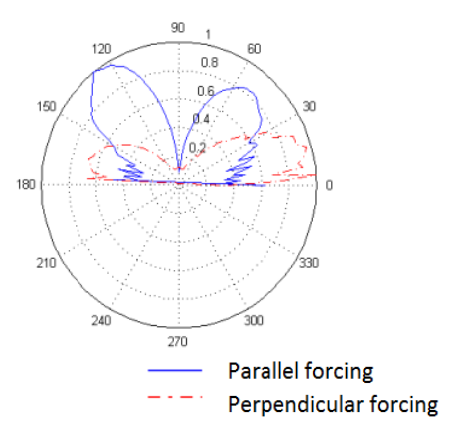

Radial component

Fig. 17 [a-c] Parallel and perpendicular forcing on $a / \lambda=0.11(3 \mathrm{~mm}) ; \theta$ component normalized with maximum scatter, $\theta$ component normalized without edge waves and radial component, respectively.

incidences being $30^{\circ}$ and $75^{\circ}$. The scattering wave patterns observed at incident angles between the extremities are a combination of parallel and perpendicular forces acting on the crack face.

The radial component of the $a / \lambda=0.11$ and 0.23 scattered wave pattern have a slightly stronger back-scatter and features of a S0 point-like source at incident angle $30^{\circ}, 45^{\circ}$ and $60^{\circ}$ incidences as shown in Figs. 13 and 16. An interesting scattering effect is the surface wave propagating on the horizontal boundary, contrasted in Figs. 5 and 6 . The $a / \lambda=0.11$ and 0.23 results showed weaker forward-scattered edge waves amplitude as shown in Fig. 8.

\subsection{Generated Wave Patterns by Tractions on Crack Faces}

In general, the simulated wave pattern investigation represents much of a point-like source and as incident angle decreases the maximum wave field amplitude increases. The applied-traction on the crack face SHO wave patterns results have shown similarity to the first investigation $\mathrm{SH} 0$ scattered wave pattern as shown in Figs. 12 and 15. However, it did not match well in the S0 wave patterns especially for larger incident angles 
as shown in Figs. 13 and 16. The $a / \lambda=0.11$ and 0.23 simulated wave pattern reported a slightly larger forward to back-scattered edge wave ratio and the SH0 wave displacements are slightly smaller compared to the original scattered pattern as shown in Figs. 11 and 14. Furthermore, perpendicular and parallel forcing components to the $3 \mathrm{~mm}$ crack face are investigated separately. Fig. 17 showed that the perpendicular forcing wave pattern appears much like a symmetrical point-like source pattern, however, the parallel forcing have shown similar scattered $75^{\circ}$ and $60^{\circ}$ wave pattern.

\section{Discussions}

The low frequency approach (the wavelength is significant larger than plate thickness and crack dimension) makes the assumption that the problem can be analyzed in plane stress condition.

One can obtain normal and shear stresses of elastic waves in a semi-infinite medium problem in plane stress conditions by using the simple change of elastic constants to convert plane strain results to plane stress, which is found in Ref. [2]. In Fig. 18, the FE and analytical incident planar wave stresses along the $\mathrm{y}$ axis have similar trend. However, the difference in stresses, especially $\sigma_{x x}$, is due to the additional contribution of edge wave propagating along the plate boundary. Since the incident wave is excited as a cylindrical source, the incident wave field includes a major component from the edge wave if excited at a smaller angle of incidence.

For all angle of incidences, the relationship between stresses and depth to wavelength have shown linear behavior until the depth is approximately $20 \%$ of wavelength which is shown in Fig. 18.

Theoretically, the simulated wave pattern will be exactly the same as the scattered wave pattern if the stresses are applied to the crack face as a function of depth. When crack length is less than $20 \%$ of wavelength, the wave pattern can simply be simulated as body-forces acting on the crack face; hence equivalent to a point-like source.

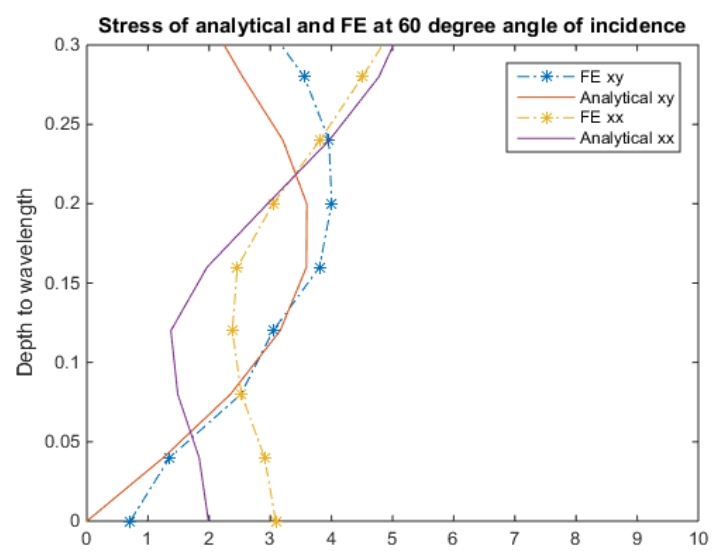

Fig. 18 Normalized analytical plane stress and FE normal and shear stresses with depth (along the $y$ axis).

The preceding FE results show that the scattered wave pattern and amplitude due to an edge crack depend on both the crack length and the incident angle. The small cracks have S0 and SH0 point-like source scattered wave patterns which are better represented in smaller incident angles. For larger incident angles, the scattered wave patterns have a stronger forward scattered lobe. These wave patterns are expected since smaller incident angles have a stronger perpendicular force component since $\sigma_{x x}$ is more dominant for smaller angle of incidences. Whereas $75^{\circ}$ and $60^{\circ}$ incidence have a relatively stronger parallel force component since $\sigma_{x y}$ has more influence for larger angle of incidences. Thus the combination of parallel and perpendicular components on the crack face can strongly influence the wave pattern.

In Fig. 9, the SH0 wave pattern is almost symmetrical when $a / \lambda$ is small, hence under this condition, the scattered wave pattern can be represented as a point-source and its amplitude is a function of $a$ as shown in Fig. 10.

The S0 scattered wave patterns have shown multiple narrow side lobes near the plate boundary, which are distinguishable in Fig. 13. These narrow lobes are interference of head waves connecting with the $\mathrm{S} 0$ and $\mathrm{SH} 0$ as seen in radial components in Figs. $5 \mathrm{~b}$ and $6 \mathrm{~b}$. In Fig. $4 \mathrm{a}$, the dispersion curve indicated a weak SH0 signal in the radial component which also suggested the existence of head waves since head 
waves have similar velocity as the SH0 wave. The $a / \lambda$ $=0.23$ polar plot showed some back-scattered multiple narrow lobes which can be seen in Fig. 10. The inaccuracy is due to assumption of constant stress profile for small crack and in order to simulate an accurate scattered wave pattern, the full stress profile is needed to be considered. The $75^{\circ}$ and $60^{\circ}$ incidences S0 scattered wave pattern results are significantly weaker and appears inaccurate in which the notch width may be a significant contributor to the scattered pattern.

\section{Conclusions}

The edge crack SO and SH0 scattered wave fields when impinged by incident S0 wave have been reported. The scattered wave amplitude for a small crack of length $a, a / \lambda<0.23$, is linearly proportional to $a$. The scattered wave pattern for a small crack can be represented as a point source consisting body-forces doublets. This investigation suggested that the scattered wave pattern and amplitudes can be utilized to characterize the edge cracks for structural health monitoring.

Further work is currently under way to further characterize and quantity the forward scattering response, as a pre-requisite for an effective approach to the inverse problem of estimating the crack size from measurement of the scattered field.

\section{References}

[1] Su, Z. Q., and Ye, L. 2009. "Lecture Notes in Applied and Computational Mechanics." In Identification of damage Using Lamb Waves: From Fundamentals to Applications. Vol. 48. London: Springer.

[2] Graff, K. F. 1970. Wave Motion in Elastic Solids. New York: Oxford University Press.

[3] Viktorov, I. A. 1970. Rayleigh and Lamb Wave: Physical Theory and Applications. New York: Plenum Press.

[4] Achenbach, J. D. 1973. Wave Propagation in Elastic Solids. London: North-Holland Publishing Company.

[5] Doherty, C., and Chiu, W. K. 2012. "Three-Dimensional Finite Element Modelling of Ultrasonic Guided Wave Scattering form Fuel Holes." Structural Health Monitoring 11 (4): 442-51.
[6] Doherty, C., and Chiu, W. K. 2012. "Scattering of Ultrasonic-Guided Waves for Health Monitoring of Fuel Weep Holes." Structural Health Monitoring 11 (1): 27-42.

[7] Nagy, P. B., Blodgett, M., and Golis, M. 1994. "Weep Hole Inspection by Circumferential Creeping Wave." NDT \& E International 27 (3): 131-42.

[8] Alleyne, D. N., Lowe, M. J. S., and Cawley, P. 1998. "The Reflection of Guided Waves from Circumferential Notches in Pipes." Journal of Applied Mechanics 65 (3): 635-41.

[9] Ogilvy, J. A., and Temple, J. A. G. 1983. "Diffraction of Elastic Waves by Cracks: Application to Time-of-Flight Inspection." Ultrasonics 21 (6): 259-69.

[10] Mendelsohn, D. A., Achenbach, J. D., and Keer, L. M. 1980. "Scattering of Elastic Waves by a Surface-Breaking Crack." Wave Motion 2 (3): 277-92.

[11] Achenbach, J. D., Keer, L. M., and Mendelsohn, D. A. 1980. "Elastodynamic Analysis of an Edge Crack." Journal of Applied Mechanics 47 (3): 551-6.

[12] Rajagopal, P. and Lowe, M. J. S. 2007. "Short Range Scattering of the Fundamental Shear Horizontal Guided Wave Mode Normally Incident at a Through-Thickness Crack in an Isotropic Plate." Journal of the Acoustical Society of America 122 (3): 1527-38.

[13] Ratassepp, M., Lowe, M. J. S., Cawley, P., and Klauson, A. 2008. "Scattering of the Fundamental Shear Horizontal Mode in a Plate When Incident at a through Crack Aligned in the Propagation Direction of the Mode." Journal of the Acoustical Society of America 124 (5): 2873-82.

[14] Aki, K., and Richards, P. G. 2002. Quantitative Seismology. 2nd ed. Vol. 1. Sanalito: University Science Book.

[15] Pavlakovic, B., Lowe, M., Alleyne, D., and Cawley, P. 1997. "Disperse: A General Purpose Program for Creating Dispersion Curves." In Review of Progress in Quantitative Nondestructive Evaluation, edited by Thompson, D. O., and Chimenti, D. E. New York: Springer.

[16] Auld, B. A. 1973. Acoustic Fields and Waves in Solids. Vol. 2. New York: Wiley.

[17] Lowe, M. J. S., and Diligent, O. 2002. "Low-Frequency Reflection Characteristics of S0 Lamb Wave from a Rectangular Notch in a Plate." Journal of the Acoustical Society of America 111 (1): 64-74.

[18] Lowe, M. J. S., Cawley, P., Kao, J. Y., and Diligent, O. 2002. "Low-Frequency Reflection Characteristics of Fundamental Antisymmetric Lamb Wave A0 from a Rectangular Notch in a Plate." Journal of the Acoustical 


\section{Small Edge Crack in an Isotropic Plate}

Society of America 112 (6): 2612-22.

[19] Alleyne, D. and Cawley, P. 1991. "A Two-Dimensional Fourier Transform Method for the Measurement of Propagating Multimode Signals." Journal of the Acoustical Society of America 89 (3): 1159-68.

[20] Bathe, K. J. 1982. "Finite Element Procedures in
Engineering Analysis.” Englewood Cliffs: Prentice-Hall.

[21] Lowe, M., and Diligent, O. 2001. "Reflection of Fundamental Lamb Modes from the Ends of Plates." In Review of Progress in Quantitative Nondestructive Evaluation, edited by Thompson, D. O., and Chimenti, D. E. Vol. 20. New York: Springer. 